\title{
Self-Tuning Vibration Control of a Rotational Flexible Timoshenko Arm Using Neural Networks
}

\author{
Minoru Sasaki, ${ }^{1}$ Toshimi Shimizu, ${ }^{2}$ Yoshihiro Inoue, ${ }^{3}$ and Wayne J. Book ${ }^{4}$ \\ ${ }^{1}$ Department of Human and Information Systems Engineering, Faculty of Engineering, Gifu University, 1-1 Yanagido, \\ Gifu 501-1193, Japan \\ ${ }^{2}$ Department of Mechanical Engineering, Faculty of Engineering, Ibaraki University, 4-12-1 Nakanarisawachou, \\ Hitachi 316-8511, Japan \\ ${ }^{3}$ Department of Mechanical and Systems Engineering, Gifu University, 1-1 Yanagido, Gifu 501-1193, Japan \\ ${ }^{4}$ School of Mechanical Engineering, Georgia Institute of Technology J. E. Love Jr. Manufacturing Building, 771 Ferst Drive, \\ Atlanta, GA 30332-0405, USA \\ Correspondence should be addressed to Minoru Sasaki, sasaki@gifu-u.ac.jp
}

Received 22 October 2011; Revised 20 February 2012; Accepted 24 February 2012

Academic Editor: Akira Ikuta

Copyright () 2012 Minoru Sasaki et al. This is an open access article distributed under the Creative Commons Attribution License, which permits unrestricted use, distribution, and reproduction in any medium, provided the original work is properly cited.

A self-tuning vibration control of a rotational flexible arm using neural networks is presented. To the self-tuning control system, the control scheme consists of gain tuning neural networks and a variable-gain feedback controller. The neural networks are trained so as to make the root moment zero. In the process, the neural networks learn the optimal gain of the feedback controller. The feedback controller is designed based on Lyapunov's direct method. The feedback control of the vibration of the flexible system is derived by considering the time rate of change of the total energy of the system. This approach has the advantage over the conventional methods in the respect that it allows one to deal directly with the system's partial differential equations without resorting to approximations. Numerical and experimental results for the vibration control of a rotational flexible arm are discussed. It verifies that the proposed control system is effective at controlling flexible dynamical systems.

\section{Introduction}

In this paper, a self-tuning vibration control of a rotational flexible arm using neural networks is presented. In the past several decades, there has been increasing interest in dynamics and control of flexible structures across a broad spectrum of engineering disciplines [1-7]. A new and exciting idea has been attracting increasing attention in many engineeringrelated areas. The idea is that the performance of flexible structures can be greatly improved by the use of active control. Flexible structures arise in several important areas of applications, for instance, antenna control, robotics, and large space structures. Satisfactory control of these systems is hampered by many difficulties related to sensing and identification.

Several approaches are possible when using neural networks for the control of these kinds of plants [8-10]. Two distinct approaches have been used to control a plant adaptively. There are direct control and indirect control. In direct control, the parameters of the controller are directly adjusted to reduce some norm of the output error. In indirect control, the parameters of the plant are estimated as the elements of a vector at any instant, and the parameter vector of the controller is chosen assuming that it represents the true value of the parameter vector. A self-tuning gain control is one of the indirect approaches for using neural networks. In indirect control, neural networks are learning to get the optimal controller gain for tracking a desired trajectory [11].

To the self-tuning control system, the control scheme consists of gain tuning neural networks and a variable-gain feedback controller. The neural networks are trained so as to make the root moment zero. In the process, the neural networks learn the optimal gain of the feedback controller. The feedback controller is designed based on Lyapunov's direct method. The feedback control of the vibration of the flexible system is derived by considering the time rate of change of 


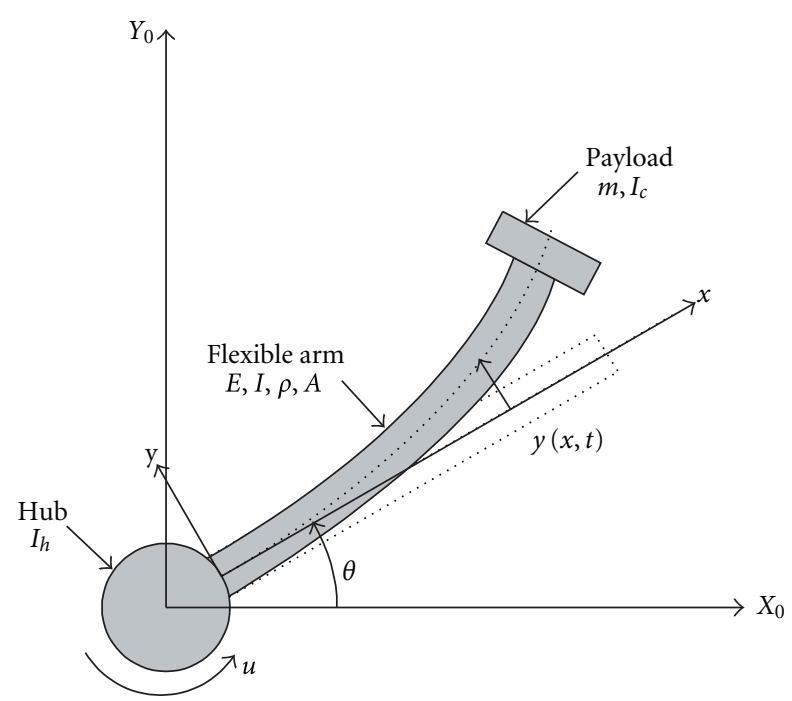

Figure 1: Rotational flexible arm.

the total energy of the system [12-18]. This approach has the advantage over the conventional methods in the respect that it allows one to deal directly with the system's partial differential equations without resorting to approximations.

Numerical and experimental results for the vibration control of a rotational flexible arm are presented. It verifies that the proposed control system is effective at controlling flexible dynamical systems.

\section{Equation of Motions and Boundary Conditions}

The rotational flexible arm depicted in Figure 1 of total length $L$, area moment of inertia $I$, cross-sectional area $A$, density $\rho$, Young's modulus $E$, shear modulus $G$, and shear coefficient $\kappa$ is attached at one end to a payload of mass $m$ and inertia $I_{c}$ and on the other end a hub of inertia $I_{h}$, which in turn is connected to an actuator that supplies a torque $u$. We obtain the following equations of motion with the boundary conditions:

$$
\begin{gathered}
\rho A\left(\frac{\partial^{2} y}{\partial t^{2}}+x \frac{\partial^{2} \vartheta}{\partial t^{2}}\right)-\kappa G A\left(\frac{\partial^{2} y}{\partial x^{2}}-\frac{\partial \phi}{\partial x}\right)=0 \\
\rho I\left(\frac{\partial^{2} \phi}{\partial t^{2}}+\frac{\partial^{2} \vartheta}{\partial t^{2}}\right)-E I \frac{\partial^{2} \phi}{\partial x^{2}}-\kappa G A\left(\frac{\partial y}{\partial x}-\phi\right)=0 \\
I_{h} \frac{\partial^{2} \vartheta}{\partial t^{2}}=E I \frac{\partial \phi(t, 0)}{\partial x}+u \\
m \frac{\partial^{2}}{\partial t^{2}}(y(L, t)+L \vartheta)=-\kappa G A\left(\frac{\partial y(L, t)}{\partial x}-\phi(L, t)\right), \\
I_{c} \frac{\partial^{2}}{\partial t^{2}}(\phi(L, t)+\vartheta)=-E I \frac{\partial \phi(L, t)}{\partial x},
\end{gathered}
$$

$$
\begin{aligned}
& y(0, t)=0, \\
& \phi(0, t)=0 .
\end{aligned}
$$

If the shear deformation and rotary inertia can be neglected, the model of the flexible arm is presented by the EulerBernoulli beam model [5].

Taking the Laplace transform of (1)-(7), we get the following:

$$
\begin{gathered}
\rho A s^{2}(Y+x \Theta)-\kappa G A\left(\frac{\partial^{2} Y}{\partial x^{2}}-\frac{\partial \Theta}{\partial x}\right)=0, \\
\rho I s^{2}(\Phi+\Theta)-E I \frac{\partial^{2} \Phi}{\partial x^{2}}-\kappa G A\left(\frac{\partial Y}{\partial x}-\Phi\right)=0, \\
I_{h} s^{2} \Theta=E I \frac{\partial \Phi(s, 0)}{\partial x}+U, \\
m s^{2}(Y(L, s)+L \Theta)=-\kappa G A\left(\frac{\partial y(L, s)}{\partial x}-\Phi(L, s)\right), \\
I_{c} s^{2}(\Phi(L, s)+\Theta)=-E I \frac{\partial \Phi(L, s)}{\partial x}, \\
Y(0, s)=0 \\
\Phi(0, t)=0 .
\end{gathered}
$$

\section{Stabilizing Feedback Control}

The problem is to find feedback control $u$ such that the arm's total energy given by

$$
\begin{aligned}
V & \frac{a_{1}}{2} \int_{0}^{L}\left[\rho A\left\{\frac{\partial y}{\partial t}+x \frac{\partial \theta}{\partial t}\right\}^{2}+\rho I\left\{\frac{\partial \phi}{\partial t}+\frac{\partial \theta}{\partial t}\right\}^{2}\right] d x \\
& +\frac{a_{2}}{2} I_{h}\left\{\frac{\partial \theta}{\partial t}\right\}^{2}+\frac{a_{3}}{2} \int_{0}^{L}\left[E I\left\{\frac{\partial \phi}{\partial x}\right\}^{2}+\kappa G A\left\{\phi-\frac{\partial y}{\partial x}\right\}^{2}\right] d x .
\end{aligned}
$$

The time rate of change of $V$ is given by

$$
\begin{aligned}
\frac{\partial V}{\partial t}=a_{1} \int_{0}^{L}\left[\rho A\left\{\frac{\partial y}{\partial t}+x \frac{\partial \theta}{\partial t}\right\}\left\{\frac{\partial^{2} y}{\partial t^{2}}+x \frac{\partial^{2} \theta}{\partial t^{2}}\right\}\right. \\
\left.+\rho I\left\{\frac{\partial \phi}{\partial t}+\frac{\partial \theta}{\partial t}\right\}\left\{\frac{\partial^{2} \phi}{\partial t^{2}}+\frac{\partial^{2} \theta}{\partial t^{2}}\right\}\right] d x \\
+a_{2} I_{h}\left\{\frac{\partial \theta}{\partial t}\right\}\left\{\frac{\partial^{2} \theta}{\partial t^{2}}\right\} \\
+a_{3} \int_{0}^{L}\left[E I\left\{\frac{\partial \phi}{\partial x}\right\}\left\{\frac{\partial^{2} \phi}{\partial x \partial t}\right\}\right. \\
\left.+\kappa G A\left\{\phi-\frac{\partial y}{\partial x}\right\}\left\{\frac{\partial \phi}{\partial t}-\frac{\partial^{2} y}{\partial x \partial t}\right\}\right] d x .
\end{aligned}
$$


Substituting (1) into (2) reduces to

$$
\begin{aligned}
\frac{\partial V}{\partial t}=a_{1} \int_{0}^{L}\left[\left\{\frac{\partial y}{\partial t}+x \frac{\partial \theta}{\partial t}\right\}\left\{\kappa G A\left(\frac{\partial^{2} y}{\partial x^{2}}-\frac{\partial \phi}{\partial x}\right)\right\}\right. \\
\left.+\left\{\frac{\partial \phi}{\partial t}+\frac{\partial \theta}{\partial x}\right\}\left\{E I \frac{\partial^{2} \phi}{\partial x^{2}}-\kappa G A\left(\phi-\frac{\partial y}{\partial x}\right)\right\}\right] d x \\
+a_{2} \frac{\partial \theta}{\partial t}\left\{E I \frac{\partial \phi(0, t)}{\partial x}+u\right\} \\
+a_{3} \int_{0}^{L}\left[E I\left\{\frac{\partial \phi}{\partial x}\right\}\left\{\frac{\partial^{2} \phi}{\partial x \partial t}\right\}\right. \\
\left.+\kappa G A\left\{\phi-\frac{\partial y}{\partial x}\right\}\left\{\frac{\partial \phi}{\partial t}-\frac{\partial^{2} y}{\partial x \partial t}\right\}\right] d x .
\end{aligned}
$$

Equation (17) is then integrated by parts, and boundary conditions (3)-(7) are substituted into the resulting equation. The result is

$$
\frac{\partial V}{\partial t}=\frac{\partial \theta}{\partial t}\left(a_{2} u+\left(a_{3}-a_{1}\right) E I \frac{\partial \phi(t, 0)}{\partial x}\right) .
$$

Taking into consideration of the fact that there is a feedback control law which gives nonpositive $\partial V / \partial t$ is

$$
u=-K_{1} \frac{\partial \theta}{\partial t}-K_{2} E I \frac{\partial \phi(t, 0)}{\partial x} \quad\left(K_{1}, K_{2}>0\right) .
$$

Substituting (19) into (18) leads to

$$
\frac{\partial V}{\partial t}=-K\left(\frac{\partial \theta}{\partial t}\right)^{2} \leq 0
$$

which is negative semidefinite. This implies that the closedloop system is energy dissipative and, hence, stable. This control law is elegant. Notice that the rigorous stability proof does not depend on introducing spatial discretization methods. Of important practical consequence, notice that controllers based on this law are easy to implement since no state estimation is required. The bending moment can be measured by using conventional strain gauges. However, the tuning of a feedback gain needs to be addressed.

\section{Self-Tuning Neural Controller}

The controllers are often poorly tuned. The reason is that it is difficult to tune a parameter by trial and error. We can embed algorithms inside computers that "learn from experience" and self-tune the controllers so as to improve closed-loop performance [16]. Often this learning process builds up a mathematical model based on experimental input/output data; this operation is known as system identification or parameter estimation. The model could be a complete transfer function or simply the gain and phase of the plant at a gain input frequency. A full process model is estimated using system identification methods, and an analytic design procedure uses the model to self-tune the coefficients of a fixed control law. A self-tuning gain control is one of the indirectly approaches for using a neural networks [5]. In

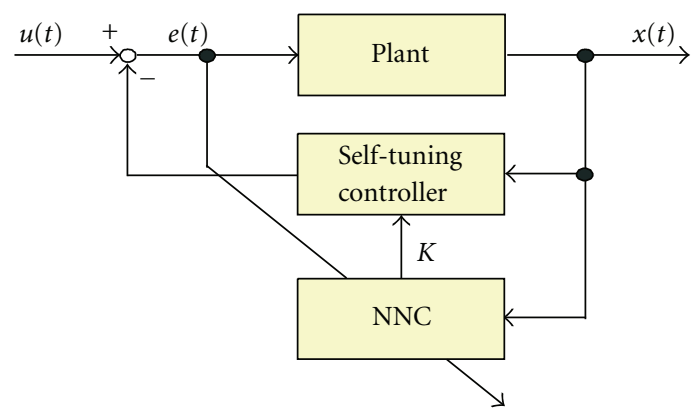

FIGURE 2: Block diagram of the proposed control system.

indirectly control as shown in Figure 2, neural network is learning to get the optimal controller gain for suppressing vibration of the flexible arm. The control scheme consists of a gain tuning neural network and a variable-gain feedback controller. In the process, the neural network learns the optimal gain of the feedback controller. The backpropagation method is a gradient descent method that establishes the weight in a multilayer, feedforward adaptive neural network. Learning is accomplished by successively adjusting the weight based on a set of input patterns and a corresponding set of desired output patterns. During this iterative process, an input pattern is presented to the network and propagated forward to determine the resulting signal at the output units. The differences between the actual resulting output signal and the predetermined desired output signal in each output unit represents an error that is backpropagated through the network in order to adjust the weights. The learning process continues until the network responds with an output signal the sum of whose root-mean square errors from the desired output signals are less than a preset value. The training process using backpropagation is a difficult process. It is necessary to find an appropriate architecture, adequate size and quality of training data, satisfactory initialization, learning parameter values, and to avoid overtraining effects. To speed up the convergence behavior, the selection of parameters such as the learning rates is done by using the utilization of a momentum factor. The learning rule utilized consists of a weight update using momentum $\alpha_{c}$ with the exception that each weight has its own learning rate parameter $\eta_{c}$. To minimize the cost function, the updating equation of the weights is defined by

$$
\Delta w_{p q}^{r}(t)=-\eta_{c} \frac{\partial E}{\partial w_{p q}^{r}(t)}+\alpha_{c} \Delta w_{p q}^{r}(t-1)
$$

where $w_{p q}^{r}$ is the weight value at $r$ th layer located between nodes $p$ and $q, t$ is the present iteration, and $w_{p q}(t)$ is the weight increment which is equal to the product of $\eta_{c}$ and the partial derivative of the objective function with respect to the weight, that is,

$$
w_{p q}^{r}(t+1)=w_{p q}^{r}(t)-\Delta w_{p q}^{r}(t)
$$


TABLE 1: System parameters.

\begin{tabular}{lccc}
\hline Parameter & Nomenclature & Values & Unit \\
\hline Length of the arm & $L$ & 1.0 & $\mathrm{~m}$ \\
Arm cross-sectional area & $A$ & $6.0 \times 10^{-4}$ & $\mathrm{~m}^{2}$ \\
Volume mass density & $\rho$ & 2.7667 & $\mathrm{Kg} / \mathrm{m}^{3}$ \\
$\begin{array}{l}\text { Young's modulus } \\
\text { Internal structural }\end{array}$ & $E$ & $6.8944 \times 10^{10}$ & $\mathrm{~Pa}$ \\
damping & $C$ & 0.0 & $\mathrm{Kg} / \mathrm{sec}$ \\
$\begin{array}{l}\text { Payload moment of } \\
\text { inertia }\end{array}$ & $I_{c}$ & 0.0 & $\mathrm{Kg} \cdot \mathrm{m}^{2}$ \\
$\begin{array}{l}\text { Payload mass } \\
\text { Shear correction factor }\end{array}$ & $m$ & 0.0 & $\mathrm{Kg}$ \\
Shear modulus & $\kappa$ & $5 / 6$ & - \\
\hline
\end{tabular}

where

$$
\begin{gathered}
E=\frac{1}{2} a x(t)^{2}+\frac{1}{2} b e(t)^{2}, \\
e(t)=u(t)-K x(t),
\end{gathered}
$$

where $x(t)$ is the output moment, $u(t)$ is the control input, and $a$ and $b$ are the weighting coefficients.

According to the generalized $\delta$-rule, neural network learning is performed for each sampling using (21) to minimize the cost function $E$. The momentum $\alpha_{c}$ is changed dynamically, because each problem has a range of optimal $\alpha_{c}$ values to avoid oscillations.

\section{Numerical Simulation and Experimental Results and Discussion}

The physical parameters of the system are presented in Table 1. A multilayer feedforward network, consisting of three layers with no inner feedback loop, was used for the self-tuning feedback controller. The size of the neuralnetwork is defined as the minimum size in which the weights between the neurons are such that the neural-network's output matches with the optimal feedback gain; however, it is hard to define that analytically. In this study, the input layer has 6 neurons, the hidden layer has 6 neurons, and the output layer has 1 neurons. Neurons in the input layer represent the reference signals $u(t)$ and $u(t-1)$, the control signals $e(t)$ and $e(t-1)$, the feedback gain $K$, and the plant outputs $x(t)$ and $x(t-1)$. The neuron in the output layer represents the feedback gain. The input neuron activation function was assumed to be the linear function $f(x)=x$, and all the thresholds were assumed to be zero. To allow for the nonlinear effect, the sigmoid function $f(x)=1 /\{1+$ $\exp (-x)\}$ was used to the hidden layer neurons. The output neuron activation function was assumed to be a function $f(x)=\ln \{1+\exp (x)\}$. The neural network controller design has been carried out on the MATLAB platform. The desired tip acceleration is widely accepted that bang-bang acceleration profiles lead to time optimal trajectories for the case of rigid body arm. We set the desired rise time and displacement from initial point to the desired end-point to $1.0[\mathrm{sec}]$ and $1.15[\mathrm{~m}]$. Figure 3 shows the gain variation of

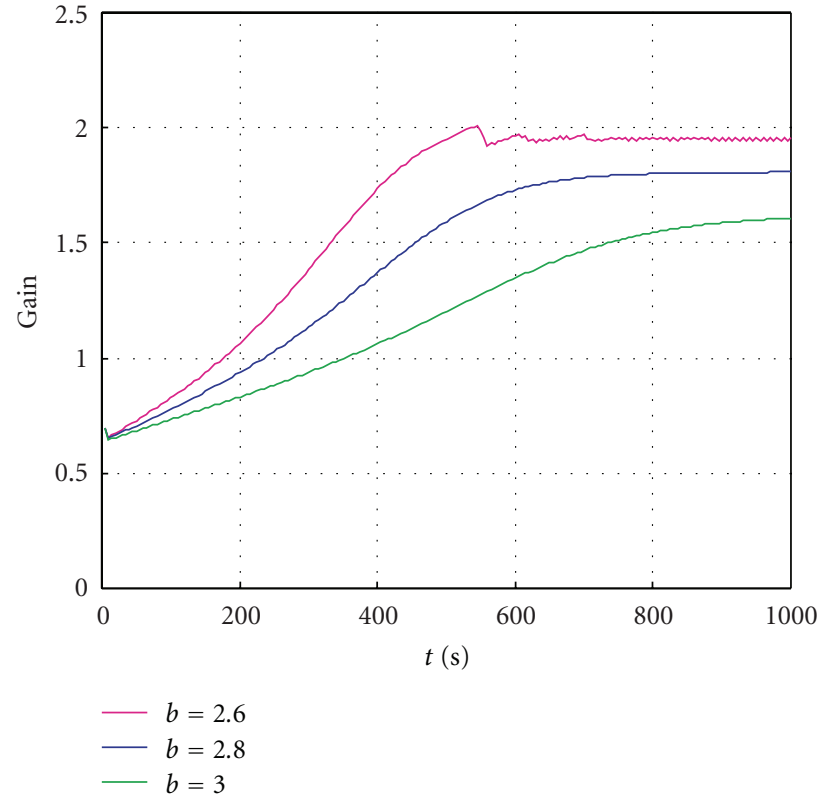

FIGURE 3: Gain variations of the self-tuning controller.

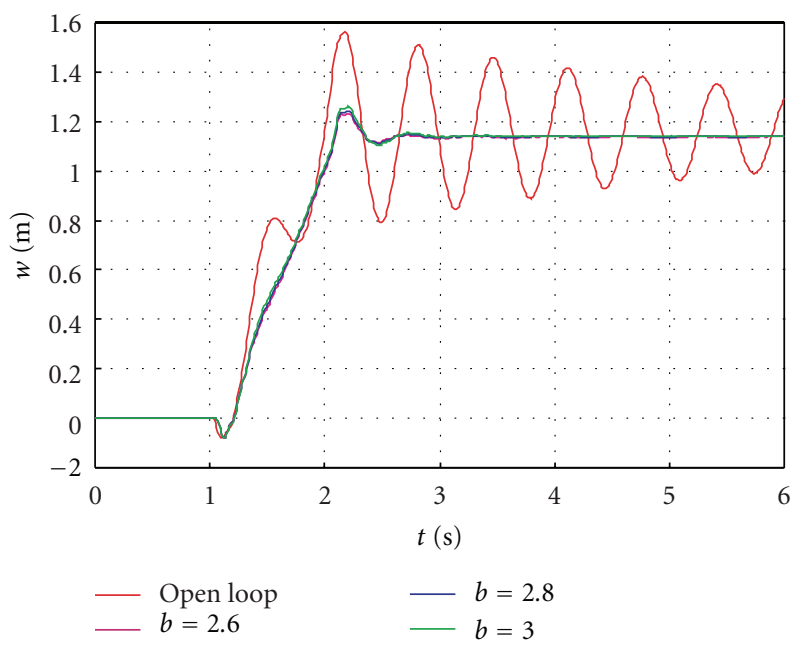

Figure 4: Time response of the tip displacement.

the self-tuning controller. Figure 4 shows the time response of the system. Figure 5 shows the learning curve of the selftuning control system. Figure 6 shows the time response of the self-tuning control system for the flexible arm with best parameter values of $\alpha_{c}$ and $\eta_{c}$. The best parameter value of $\alpha_{c}$ is 0.6 and $\eta_{c}$ is 0.8 . The weighting constants $a$ and $b$ are fixed to 1 and 2.6, respectively. It can be clearly seen that the vibration suppressing performance of the self-tuning control system using neural networks is better than that without control.

In the experiment, same neural network is used as the numerical simulation. For the implementation of fast controllers, we routinely use the TMS320C31-based digital signal processing system (DSP-CIT) along with a set of design 


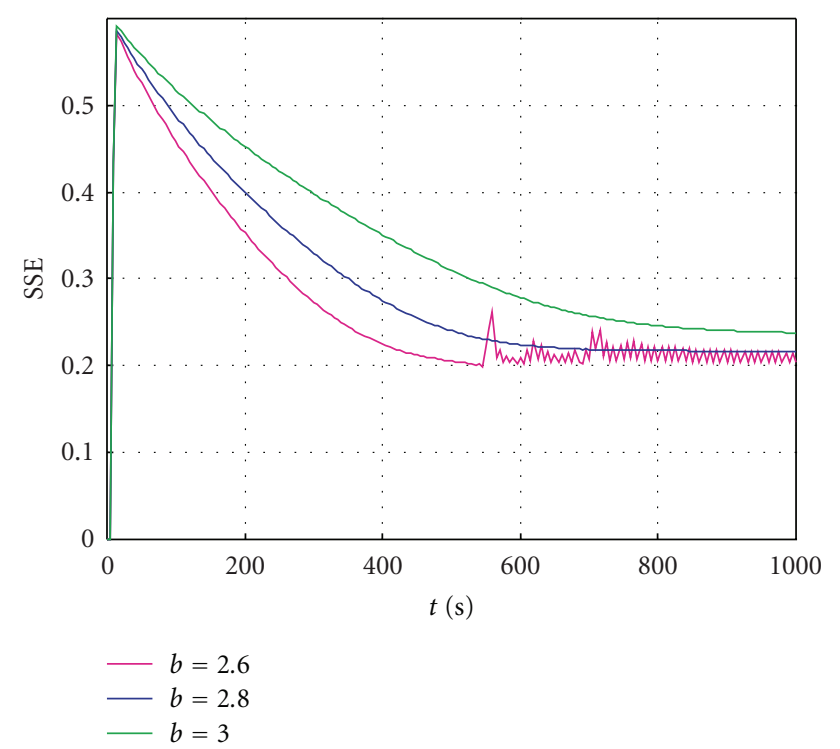

FIGURE 5: Learning curve of the self-tuning system.

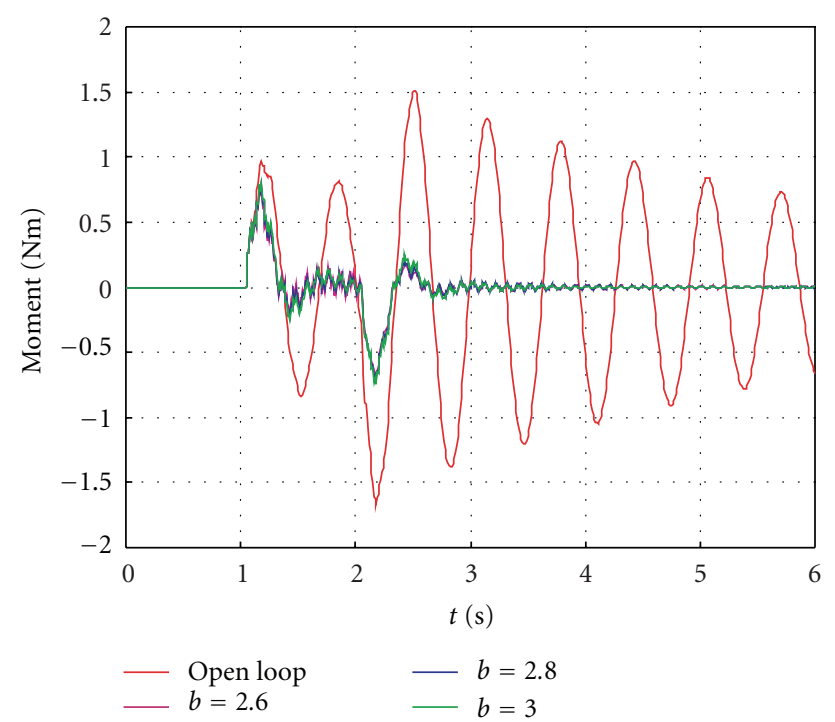

FIgURE 6: Time response of the root moment.

and implementation software tools, including an automatic code generator. The design in the analogue domain was carried out, and the controller was discretized, after checking for the effects of discretization, computational delays, AD- and DA quantization, the signal processor code was generated and downloaded. The sampling period was 10 [ms]. Figure 7 is the experimental setup for implementation and assessment of the neural network control system. Figure 8 shows the experimental results of the root moment. Figure 9 shows the input voltage of the motor. This control system can suppress the vibrations of the flexible arm within a short time in comparison with no moment feedback. From these results it is concluded that using the neural network may be possible in some cases to tune an optimal gain of the feedback controller.

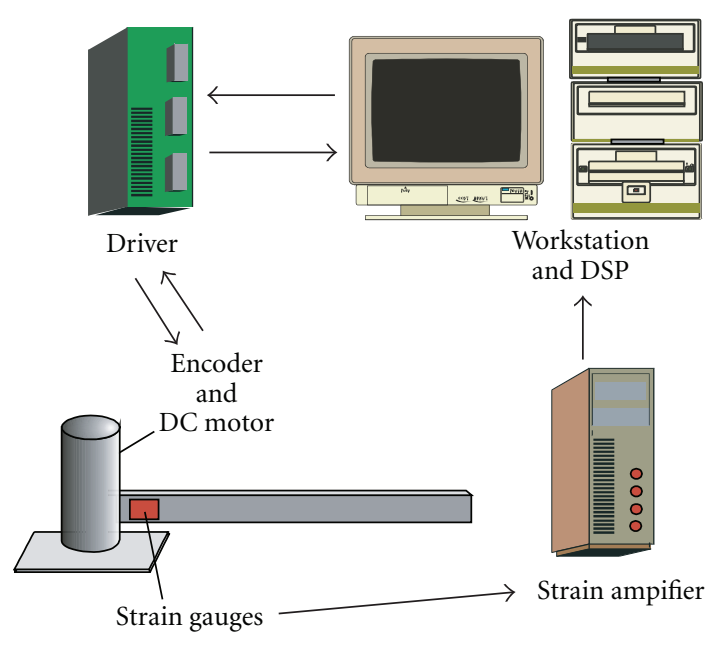

Figure 7: Experimental setup.

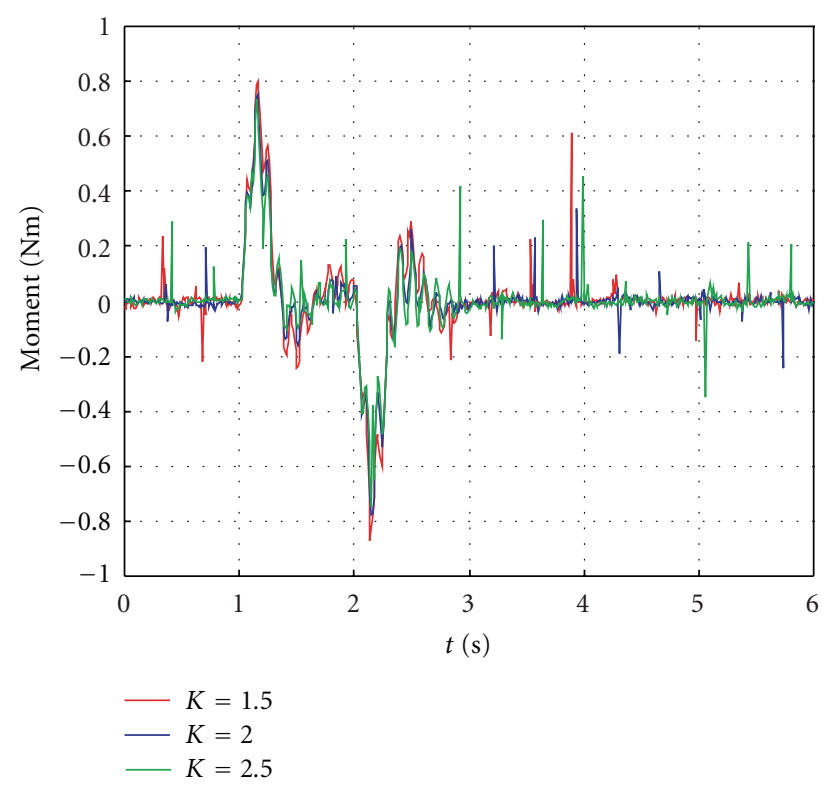

Figure 8: Time response of the root moment.

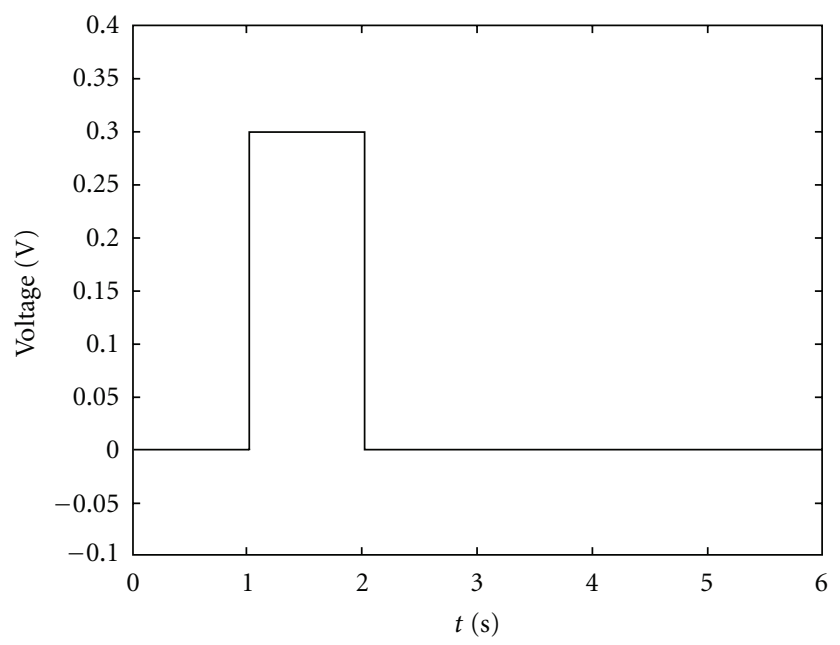

FIGURE 9: Input voltage of the motor. 


\section{Conclusions}

A self-tuning control system of a rotational flexible arm using neural networks was presented. The neural networks using new cost function learned optimal feedback gain to avoid excessive control input. Numerical and experimental results show that the proposed controller is useful for vibration control of the rotational flexible arm.

\section{Appendix}

In the case of the rotational Timoshenko arm, the resulting solution to (8), (9), and (10) is given by

$$
\begin{aligned}
Y(x, s)= & \frac{\Delta k_{1}}{\Delta} e^{\lambda_{1} x}+\frac{\Delta k_{2}}{\Delta} e^{\lambda_{2} x}-\frac{\Delta k_{3}}{\Delta} e^{-\lambda_{1} x}-\frac{\Delta k_{4}}{\Delta} e^{-\lambda_{2} x}-x \Theta \\
\Phi(x, s)= & \left(\lambda_{1}-\frac{\rho s^{2}}{\lambda_{1} \kappa G}\right)\left(k_{1} e^{\lambda_{1} x}-k_{3} e^{-\lambda_{1} x}\right) \\
& +\left(\lambda_{2}-\frac{\rho s^{2}}{\lambda_{2} \kappa G}\right)\left(k_{2} e^{\lambda_{2} x}-k_{4} e^{-\lambda_{2} x}\right)-\Theta
\end{aligned}
$$

where

$$
\begin{aligned}
a_{11}= & \left\{m s^{2}+\frac{\rho A s^{2}}{\lambda_{1}}\right\} e^{\lambda_{1} L}, \\
a_{12}= & \left\{m s^{2}+\frac{\rho A s^{2}}{\lambda_{2}}\right\} e^{\lambda_{2} L}, \\
a_{13}= & \left\{m s^{2}-\frac{\rho A s^{2}}{\lambda_{1}}\right\} e^{-\lambda_{1} L}, \\
a_{14}= & \left\{m s^{2}-\frac{\rho A s^{2}}{\lambda_{2}}\right\} e^{-\lambda_{2} L}, \\
a_{21}= & \left(\lambda_{1}-\frac{\rho s^{2}}{\kappa G \lambda_{1}}\right)\left(I_{c} s^{2}+E I(1+C s) \lambda_{1}\right) e^{\lambda_{1} L}, \\
a_{22}= & \left(\lambda_{2}-\frac{\rho s^{2}}{\kappa G \lambda_{2}}\right)\left(I_{c} s^{2}+E I(1+C s) \lambda_{2}\right) e^{\lambda_{2} L}, \\
a_{43} & -\left\{I_{h} s^{2}\left(\lambda_{1}-\frac{\rho s^{2}}{\kappa G \lambda_{1}}\right)+E I(1+C s)\left(\lambda_{1}^{2}-\frac{\rho s^{2}}{\kappa G}\right)\right\}, \\
a_{41} & =\left\{I_{h} s^{2}\left(\lambda_{1}-\frac{\rho s^{2}}{\kappa G \lambda_{1}}\right)-E I(1+C s)\left(\lambda_{1}^{2}-\frac{\rho s^{2}}{\kappa G}\right)\right\} \\
\left.a_{31}-\frac{\rho s^{2}}{\kappa G \lambda_{1}}\right)\left(I_{c} s^{2}-E I(1+C s) \lambda_{1}\right) e^{-\lambda_{1} L}, & a_{32}=a_{33}=a_{34}=1, \\
a_{24} & \left.=-\left(\lambda_{2}-\frac{\rho s^{2}}{\kappa G}\right)\right\} \\
& =\left(I_{c} s^{2}-E I(1+C s) \lambda_{2}\right) e^{-\lambda_{2} L},
\end{aligned}
$$

$$
\begin{aligned}
& a_{44}=-\left\{I_{h} s^{2}\left(\lambda_{2}-\frac{\rho s^{2}}{\kappa G \lambda_{2}}\right)+E I(1+C s)\left(\lambda_{2}^{2}-\frac{\rho s^{2}}{\kappa G}\right)\right\} \text {, } \\
& \Delta=\left[\begin{array}{llll}
a_{11} & a_{12} & a_{13} & a_{14} \\
a_{21} & a_{22} & a_{23} & a_{24} \\
a_{31} & a_{32} & a_{33} & a_{34} \\
a_{41} & a_{42} & a_{43} & a_{44}
\end{array}\right], \\
& \Delta k_{1}=\left[\begin{array}{cccc}
0 & a_{12} & a_{13} & a_{14} \\
0 & a_{22} & a_{23} & a_{24} \\
0 & a_{32} & a_{33} & a_{34} \\
U & a_{42} & a_{43} & a_{44}
\end{array}\right], \\
& \Delta k_{2}=\left[\begin{array}{cccc}
a_{11} & 0 & a_{13} & a_{14} \\
a_{21} & 0 & a_{23} & a_{24} \\
a_{31} & 0 & a_{33} & a_{34} \\
a_{41} & U & a_{43} & a_{44}
\end{array}\right], \\
& \Delta k_{3}=\left[\begin{array}{cccc}
a_{11} & a_{12} & 0 & a_{14} \\
a_{21} & a_{22} & 0 & a_{24} \\
a_{31} & a_{32} & 0 & a_{34} \\
a_{41} & a_{42} & U & a_{44}
\end{array}\right], \\
& \Delta k_{4}=\left[\begin{array}{cccc}
a_{11} & a_{12} & a_{13} & 0 \\
a_{21} & a_{22} & a_{23} & 0 \\
a_{31} & a_{32} & a_{33} & 0 \\
a_{41} & a_{42} & a_{43} & U
\end{array}\right], \\
& \lambda= \pm\left[\frac { 1 } { 2 } \left\{\left(\frac{1}{E(1+C s)}+\frac{1}{\kappa G}\right) \rho s^{2}\right.\right. \\
& \pm\left(\frac{\rho^{2} s^{4}}{E^{2}(1+C s)^{2}}-\frac{2 \rho^{2} s^{4}}{E(1+C s) \kappa G}\right. \\
& \left.\left.\left.+\frac{\rho^{2} s^{4}}{\kappa^{2} G^{2}}-\frac{4 \rho A s^{2}}{E I(1+C s)}\right)^{1 / 2}\right\}\right]^{1 / 2}, \quad \lambda= \pm \lambda_{1}, \pm \lambda_{2} .
\end{aligned}
$$

\section{References}

[1] K. Yuan and C. M. Hu, "Nonlinear modeling and partial linearizing control of a slewing Timoshenko-beam," Journal of Dynamic Systems, Measurement and Control, Transactions of the Asme, vol. 118, no. 1, pp. 75-83, 1996.

[2] M. Tadi, "Comparison of two finite-element schemes for feedback control of a Timoshenko beam," in Proceedings of the 1997 ASME International Mechanical Engineering Congress and Exposition, pp. 587-596, November 1997.

[3] M. W. D. White and G. R. Heppler, "Vibration of a rotating Timoshenko beam," Journal of Vibration and Acoustics, Transactions of the Asme, vol. 118, no. 4, pp. 606-613, 1996.

[4] M. J. Balas, "Direct velocity feedback control of large space structures," Journal of Guidance, Control, and Dynamics, vol. 2, no. 3, pp. 252-253, 1979.

[5] J. Moreno-Valenzuela, "Design of output feedback tracking controllers for euler-lagrange systems by using a lyapunov function-based procedure," in Proceedings of the 43rd IEEE Conference on Decision and Control (CDC'04), pp. 4051-4056, December 2004. 
[6] Z. H. Luo, "Direct strain feedback control of flexible robot arms: new theoretical and experimental results," IEEE Transactions on Automatic Control, vol. 38, no. 11, pp. 1610-1622, 1993.

[7] B. Paden, D. Chen, R. Ledesma, and E. Bayo, "Exponentially stable tracking control for multijoint flexible-link manipulators," Journal of Dynamic Systems, Measurement and Control, Transactions of the Asme, vol. 115, no. 1, pp. 53-59, 1993.

[8] G. G. Rigatos, "Model-based and model-free control of flexible-link robots: a comparison between representative methods," Applied Mathematical Modelling, vol. 33, no. 10, pp. 3906-3925, 2009.

[9] B. Subudhi and A. S. Morris, "Soft computing methods applied to the control of a flexible robot manipulator," Applied Soft Computing Journal, vol. 9, no. 1, pp. 149-158, 2009.

[10] E. M. Nebot, G. Sentoni, and F. Masson, "125 Identification of a flexible manipulator using neural networks," Control Engineering Practice, vol. 2, no. 5, pp. 583-586, 1994.

[11] M. Kawafuku, M. Sasaki, and S. Kato, "Self-tuning pid control of a flexible micro-actuator using neural networks," in Proceedings of the IEEE International Conference on Systems, Man, and Cybernetics, pp. 3067-3072, October 1998.

[12] J. LaSalle and S. Lefschetz, Stability by Lyapunov's Direct Method with Applications, Academic Press, New York, NY, USA, 1961.

[13] R. Ortega, A. Loria, P. J. Nicklasson, and H. Sira-Ramirez, Passivity-based Control of Euler-Lagrange Systems, Springer, London, UK, 1998.

[14] M. Sasaki, K. Okuda, R. Okamoto, and F. Fujisawa, "Twodegree-of-freedom control system for motion control of flexible cartesian robots," in Proceedings of the Active Control of Vibration and Noise International Congress and Exposition (ASME '96), pp. 127-134, November 1996.

[15] M. Sasaki, T. Ueda, Y. Inoue, and W. J. Book, "Passivity-based control of rotational and translational Timoshenko arms," Advances in Acoustics and Vibration, vol. 2012, Article ID 174816, 6 pages, 2012.

[16] M. Sasaki, H. Asai, M. Kawafuku, and Y. Hori, "Self-tuning control of a translational flexible arm using neural networks," in Proceedings of the IEEE International Conference on Systems, Man, and Cybernetics, pp. 3259-3264, Nashville, Tenn, USA, October 2000.

[17] F. Zhang, D. M. Dawson, M. S. de Queiroz, and P. Vedagarbha, "Boundary control of the Timoshenko beam with free-end mass/inertial dynamics," in Proceedings of the 36th Conference on Decision and Control, pp. 245-250, San Diego, Calif, USA, December 1997.

[18] O. Morgul, "Dynamic boundary control of the Timoshenko beam," Automatica, vol. 28, no. 6, pp. 1225-1260, 1992. 

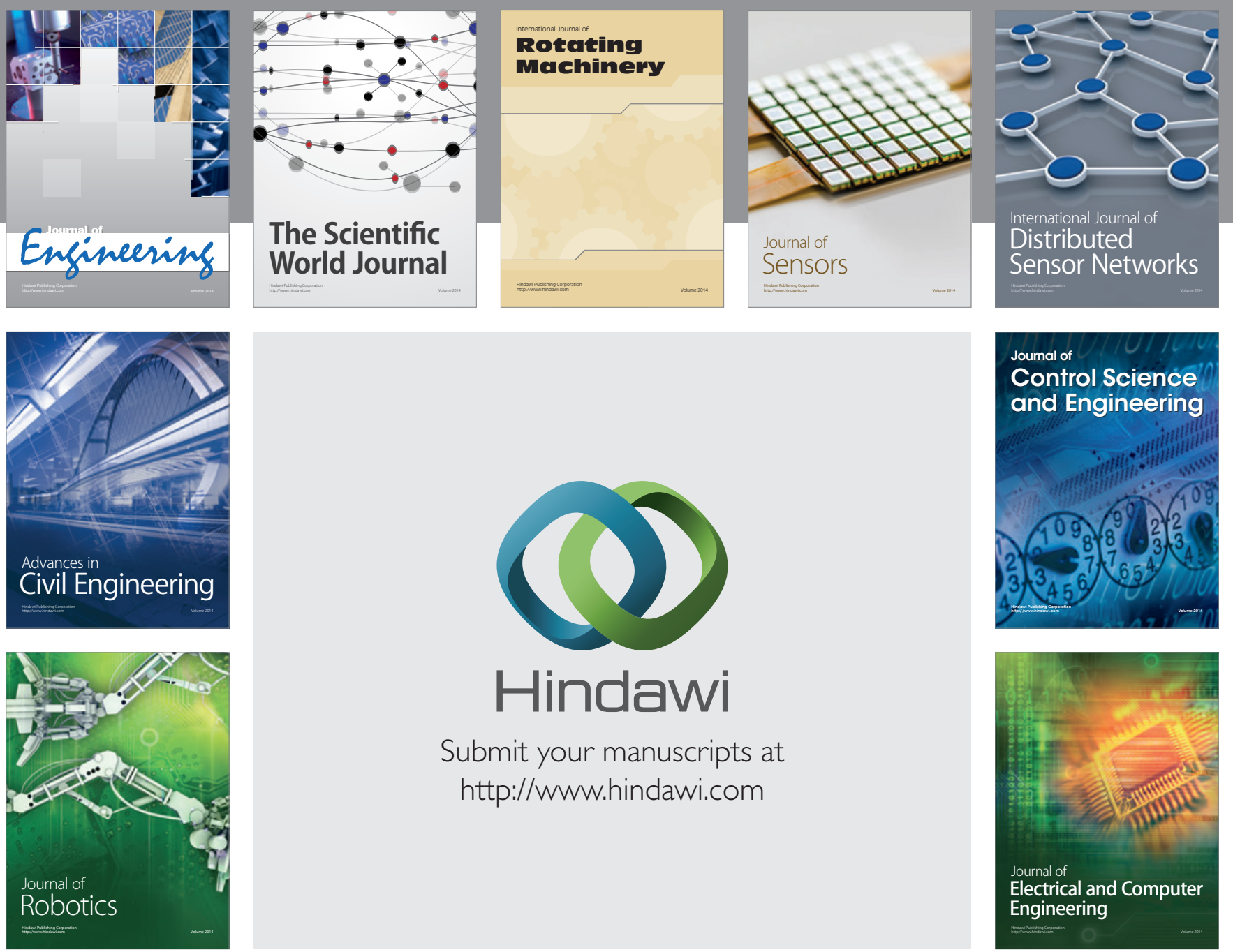

Submit your manuscripts at

http://www.hindawi.com
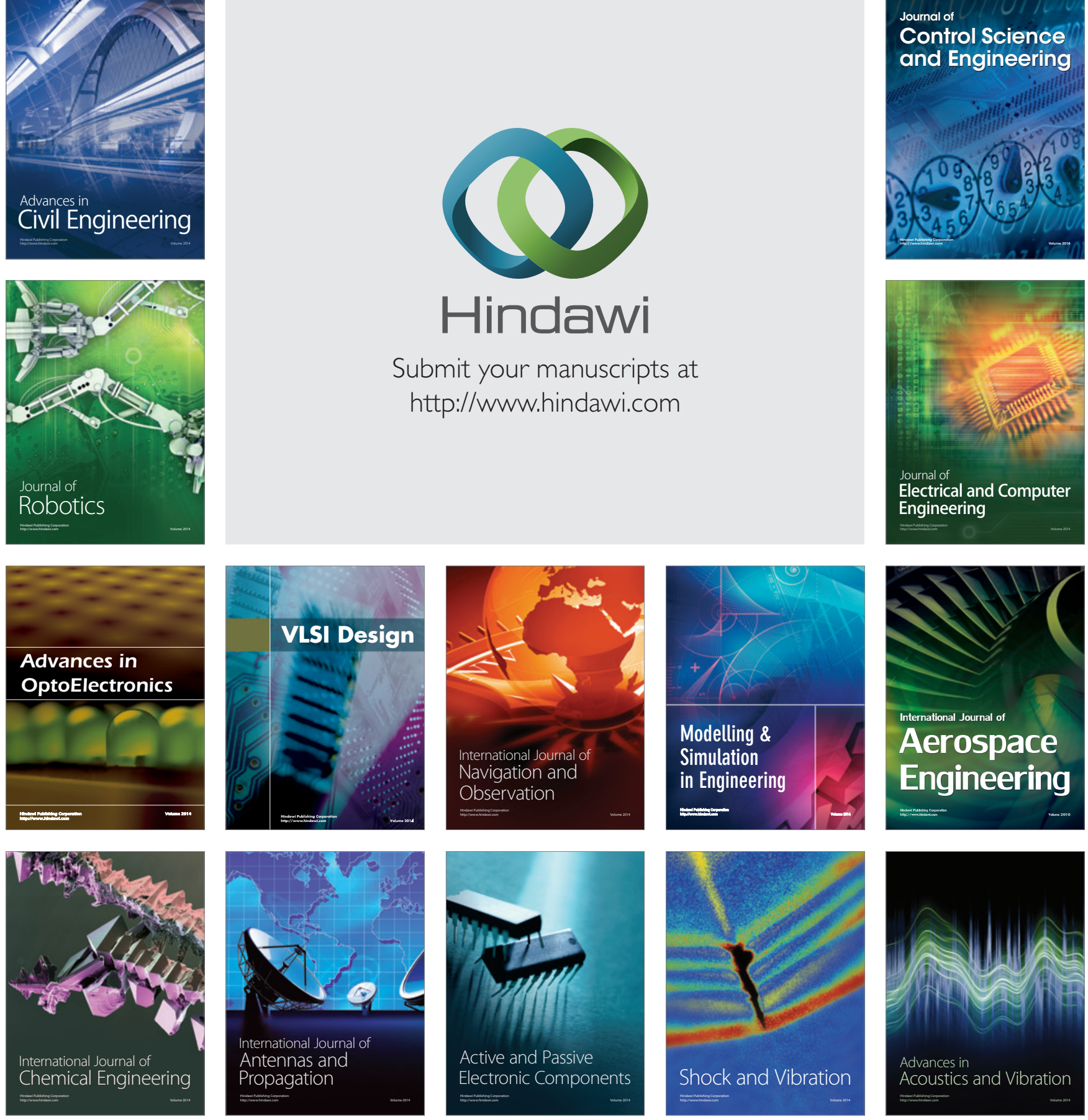